\title{
Application of Employment to College Students Based on AHP Virtual Platform
}

\author{
Huazhen Chang \\ College of Science, Northeast Dianli University, Jilin 132012, China
}

\begin{abstract}
The paper proposed an application method on college student employment using the AHP virtual platform. The application method is a simple, flexible and practical multi criterion decision method. It has been proved by experiment that the results obtained from using the Analytic Hierarchy Process analyzing multiple factors on college students employment agreed with actual data. The method is easily applicable and can provide useful information on college student employment for those who are interested.
\end{abstract}

Keywords: AHP, Consistency Check, Comparative Matrix, Weight vectors.

\section{INTRODUCTION}

Analytic Hierarchy Process (AHP) is a simple, flexible and practical multi criterion decision-making method proposed by American operational researcher T.L.Saaty in 70s. Based on AHP virtual platform, one can apply analytical hierarchy process to perform college student employment analysis. The purpose of the method is to provide an objective and systematic way of analyzing the problem for students and others who are interested.

\section{AHP VIRTUAL PLATFROM}

The Analytic Hierarchy Process is basically the same as the way of thinking for one to solve a complicated decision problem.

\section{A. Steps of Applying the AHP Method}

The basic steps are:

Stepl: Divide the problem into three layers, define the top one to be the target layer; the bottom to be the solution layer; the middle layer to be the criterion layer. The relationship between each layer is presented by lines connecting them.

Step2: Compare the weight of each criterion on the target, the weight of each plan on its corresponding criterion. The weights are commonly fixed in a way of thinking. AHP will give an algorithm to evaluate these weights.

Step3: Synthesis the two weights mentioned in step 2, and find the final weight of each criterion to the target. AHP will give an algorithm of calculation.

Step4: Make the decision based on the weights of each criterion to the target.

\section{B. Comparative Weight Vector and Comparative Pair Matrix}

Assume there are n factors $C_{1}, C_{2}, \cdots, C_{n}$ in one layer to be compared with some factor $O$ in the upper layer. Let's say there are five criteria, pick 2 random factor $C_{i}$ and $C_{j}$, use $a_{i j}$ to present the ratio of effects of $C_{i}$ and $C_{j}$ cast on $O$. All the comparative results can form a comparative pair matrix:

$$
A=\left(a_{i j}\right)_{n \times n}, a_{i j}>0, a_{j i}=\frac{1}{a_{i j}}
$$

From the characters of $a_{i j}$ given in formula (1), A is a straight reciprocal matrix, and $a_{i i}=1$.We use $c_{1}, c_{2}, \cdots, c_{5}$ to denote the 5 criteria. Using the comparative method we have the matrix ( $C_{5}^{2}$ compares were made):

$$
A=\left(\begin{array}{ccccc}
1 & 1 / 2 & 4 & 3 & 3 \\
2 & 1 & 7 & 5 & 5 \\
1 / 4 & 1 / 7 & 1 & 1 / 2 & 1 / 3 \\
1 / 3 & 1 / 5 & 2 & 1 & 1 \\
1 / 3 & 1 / 5 & 3 & 1 & 1
\end{array}\right)
$$

From the matrix A given in formula (2), since the ratio of $C_{1}$ and $C_{2}$ is $1: 2$, and the ratio of $C_{1}$ and $C_{3}$ is $4: 1$, then by the mathematic nature, the ratio of $C_{2}$ and $C_{3}$ should be $8: 1$, other than $7: 1$. This shows that results of each compare are inconsistent with each other. However, n factors will need $\frac{n(n-1)}{2}$ compares. Now, we denote $a_{i j}=w_{i} / w_{j}$ in the new matrix. We'll have 


$$
A=\left(\begin{array}{cccc}
\frac{w_{1}}{w_{1}} & \frac{w_{1}}{w_{2}} & \cdots & \frac{w_{1}}{w_{n}} \\
\frac{w_{2}}{w_{1}} & \frac{w_{2}}{w_{2}} & \cdots & \frac{w_{2}}{w_{n}} \\
\cdots & \cdots & \cdots & \cdots \\
\frac{w_{n}}{w_{1}} & \frac{w_{n}}{w_{2}} & \cdots & \frac{w_{n}}{w_{n}}
\end{array}\right)
$$

Obviously, these compares are consistent with each other, the weight of $\mathrm{n}$ factors to the object can be denoted by $\quad w=\left(w_{1}, w_{2}, \cdots, w_{n}\right)^{T}$. Obviously, we have $\sum_{i=1}^{n} w_{i}=1$. Clearly, in the matrix $A$ shown in formula (3), each row vector differs from $w$ in only one ratio element.

Generally, if a straight reciprocal matrix satisfies:

$$
a_{i j} \cdot a_{j k}=a_{i k}, i, j, k=1,2, \cdots, n .
$$

Then we call it a consistent matrix. Apparently, matrix $A$ is a consistent matrix.

If the matrix we got from comparing the weight of factors is a consistent matrix, like matrix $A$, it's natural to take the normalized eigenvector of eigenvalue $n$ to present the weights on object $O$ from multiple factors $C_{1}, c_{2}, \cdots, c_{n}$, which is then called weight vector. If the matrix is not a consistent matrix, but the inconsistency is within a tolerant range (the definition of the tolerant range will be given later), Saaty, et al. suggests that one should take the normalized eigenvector of the largest eigenvalue of A as the weight vector, which is denoted as $w$, i.e. $w$ satisfies:

$$
A w=\lambda w .
$$

The method introduced is the eigenvalue method for finding the weight of comparative matrix.

\section{Comparative Standard}

When comparing the effect of two different factors $C_{i}$ and $C_{j}$ on some object $O$ in the top layer, we need to figure out a standard to measure $a_{i j}$. Satty, et al. proposed a 1-9 standard, i.e. the value of $a_{i j}$ is chosen between integers $1,2,3,4 . .9$, which is shown in table 1 .

TABLE 1 MEAning OF $a_{i j}$ IN THE $1-9$ STANDARD

\begin{tabular}{l|l}
\hline Standard $a_{i j}$ & Meaning \\
\hline 1 & $C_{i}$ and $C_{j}$ has the same effect \\
3 & $\begin{array}{l}\text { Effect of } C_{j} \text { is slightly higher } \\
\text { Effect of } C_{j} \text { is mildly higher } \\
5\end{array}$ \\
$\begin{array}{l}\text { Effect of } C_{j} \text { is significantly higher } \\
\text { Effect of } C_{j} \text { is absolutely higher } \\
9\end{array}$ & $\begin{array}{l}\text { Effect of } C_{i} \text { is between its adjacent level higher } \\
\text { than } C_{j}\end{array}$ \\
$2,4,6,8$ & $\begin{array}{l}\text { The ratio of effeteness of } C_{j} \text { and } C_{i} \text { is } \\
\text { reversely related as the value above }\end{array}$ \\
\hline
\end{tabular}

Currently in Analytical Hierarchy Process applications, most people would use the 1-9 standards.

\section{Consistency Check on Comparative Pair Matrix}

Comparative Pair Matrix are generally not consistent matrix, but as mentioned previously, such matrix will need a consistent check to make sure the inconsistency of such matrix is within the tolerant rage. We'll discuss how to define the range in this section.

We'll use the value of $\lambda-n$ to measure the inconsistency of matrix A. Saaty et al. defines:

$$
C I=\frac{\lambda-n}{n-1}
$$

as the consistency indicator. When $C I=0, A$ is consistent matrix. However, the value of $C I$ needn't to grow with the value of $A$. Therefore, Saaty introduced another indicator $R I$ to be the Random Indicator, which is shown in table 2.

\section{TABLE 2 VALUE FOR THE RANDOM INDICATOR}

\begin{tabular}{l|lllllllllll}
\hline$n$ & 1 & 2 & 3 & 4 & 5 & 6 & 7 & 8 & 9 & 10 & 11 \\
\hline$R I$ & 0 & 0 & 0.58 & 0.90 & 1.12 & 1.24 & 1.32 & 1.41 & 1.45 & 1.49 & 1.51 \\
\hline
\end{tabular}

For those matrix which has a value of $\mathrm{n}$ greater than 3 , we'll take ratio of the consistency indicator value and the corresponding random indicator value. We name the factor the Consistency Ratio, $C R$. When the inconsistency of matrix A is out of tolerant. We'll then use the eigenvector corresponding to its maximum eigenvalue as the weight vector. Otherwise, one should repeat the comparison in order to adjust the matrix $A$.
The check on matrix $A$ using equation (6) and (7) and table 2 is called the consistency check.

$$
C R=\frac{C I}{R I}<0.1
$$




\section{The ApPlicAtion OF AHP On COLLEge StUdent EMPLOYMENT}

Since different job emphasize on different aspects, the indicator weight also differs from job to job. A precise, reasonable weight could help employees focusing on the completion of evaluating the important and complicated indicators. It may also help the employee to view the job in a more objective point of view. Here's an example of using the AHP method to analyze a college student employment problem. First we'll use the AHP method to build a comparative pair matrix, then do the consistency check and give a reasonable solution to the problem.

\section{A. Setup of the Factor Evaluating System}

Use the AHP method to analyze the trend of employment of a specific occupation. Accordingly, we'll build up a 3-layer model, including the target layer, criterion layer, and the solution layer.

The criterion layer is the students' basic needs to the employment unit, which is relatively defined. Different occupation will have different requirements. According to investigation on the subject, we have the following 7 different criteria: 1. Playing Strength, 2.Promotion Opportunities 3.Income 4.Working Environment 4.Working Strength 5. Study Opportunities 6.Innovation Degree. The following chart shows:

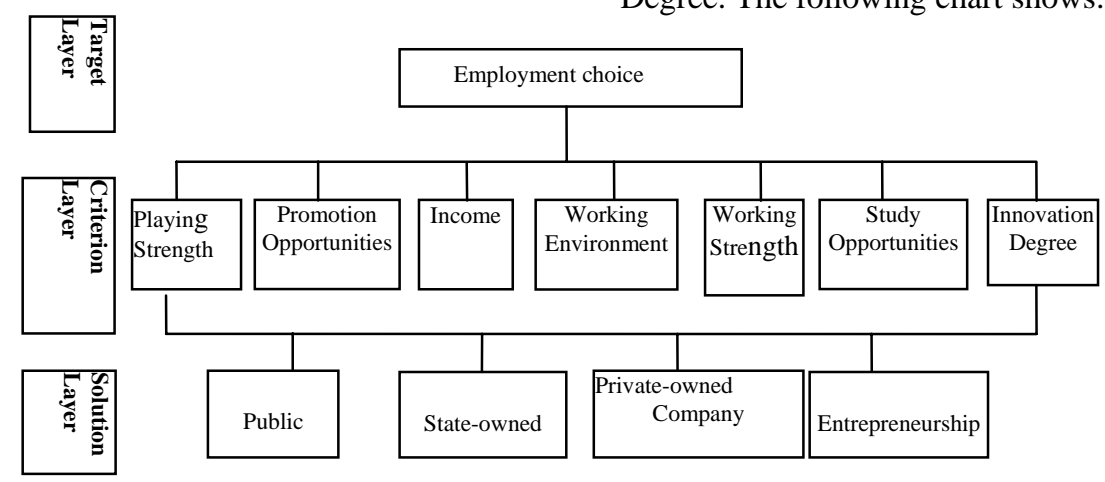

B. Building the Comparative Pair Matrix, computing the weight and Consistency Check
The results are shown in the following matrixes and table 3.

$$
\begin{aligned}
& A=\left[\begin{array}{ccccccc}
1 & 1 / 4 & 1 / 4 & 1 / 3 & 2 & 1 & 1 \\
4 & 1 & 1 & 2 & 6 & 4 & 4 \\
5 & 1 & 1 & 2 & 6 & 4 & 4 \\
3 & 1 / 2 & 1 / 2 & 1 & 5 & 3 & 3 \\
1 / 2 & 1 / 6 & 1 / 6 & 1 / 5 & 1 & 1 / 2 & 1 / 2 \\
1 & 1 / 4 & 1 / 4 & 1 / 3 & 2 & 1 & 1 \\
1 & 1 / 4 & 1 / 4 & 1 / 3 & 2 & 1 & 1
\end{array}\right] \\
& B_{1}=\left[\begin{array}{cccc}
1 & 1 / 2 & 2 & 1 / 2 \\
2 & 1 & 4 & 1 \\
1 / 2 & 1 / 4 & 1 & 1 / 4 \\
2 & 1 & 4 & 1
\end{array}\right] \\
& B_{2}=\left[\begin{array}{llll}
1 & 2 & 2 & 4 \\
1 / 2 & 1 & 1 & 2 \\
1 / 2 & 1 & 1 & 2 \\
1 / 4 & 1 / 2 & 1 / 2 & 1
\end{array}\right] \\
& B_{3}=\left[\begin{array}{cccc}
1 & 1 & 1 / 4 & 1 / 4 \\
1 & 1 & 1 / 4 & 1 / 4 \\
4 & 4 & 1 & 1 \\
4 & 4 & 1 & 1
\end{array}\right] \\
& B_{4}=\left[\begin{array}{cccc}
1 & 1 / 2 & 1 / 2 & 1 / 4 \\
2 & 1 & 1 & 1 / 2 \\
2 & 1 & 1 & 1 / 2 \\
4 & 2 & 2 & 1
\end{array}\right] B_{5}=\left[\begin{array}{cccc}
1 & 1 / 5 & 1 / 2 & 1 \\
5 & 1 & 3 & 3 \\
2 & 1 / 3 & 1 & 2 \\
1 & 1 / 3 & 1 / 2 & 1
\end{array}\right] \\
& B_{6}=\left[\begin{array}{cccc}
1 & 1 / 5 & 1 / 3 & 1 / 2 \\
5 & 1 & 2 & 4 \\
3 & 1 / 2 & 1 & 2 \\
2 & 1 / 4 & 1 / 2 & 1
\end{array}\right] \\
& B_{7}=\left[\begin{array}{cccc}
1 & 7 & 9 & 7 \\
1 / 7 & 1 & 5 & 3 \\
1 / 9 & 1 / 5 & 1 & 1 / 5 \\
1 / 7 & 1 / 3 & 5 & 5
\end{array}\right]
\end{aligned}
$$

TABLE 3

\begin{tabular}{l|l|l|l|l|l|l|c}
\hline$k$ & 1 & 2 & 3 & 4 & 5 & 6 & 7 \\
\hline$\varpi_{k 1}$ & 0.182 & 0.444 & 0.100 & 0.111 & 0.221 & 0.116 & 0.679 \\
\hline$\varpi_{k 2}$ & 0.364 & 0.222 & 0.100 & 0.222 & 0.101 & 0.532 & 0.179 \\
\hline$\varpi_{k 3}$ & 0.091 & 0.222 & 0.400 & 0.222 & 0.432 & 0.221 & 0.038 \\
\hline$\varpi_{k 4}$ & 0.364 & 0.111 & 0.400 & 0.444 & 0.255 & 0.131 & 0.104 \\
\hline
\end{tabular}




\begin{tabular}{|l|l|l|c|c|c|c|c}
\hline$\lambda_{\max }$ & 4.000 & 4.000 & 4.000 & 4.000 & 4.012 & 4.049 & 4.058 \\
\hline$C I_{k}$ & 0 & 0 & 0 & 0 & 0.007 & 0.016 & 0.019 \\
\hline$R I_{k}$ & 0 & 0 & 0 & 0 & 0.008 & 0.018 & 0.022 \\
\hline
\end{tabular}

The weight to the target of matrix $B_{1}$ is 0.252 . The weight to the target of matrix $B_{2}$ is 0.211 . The weight to the target of matrix $B_{3}$ is 0.316 . The weight to the target of matrix $B_{4}$ is 0.291 .All in all, the weight vector of the criterion layer the target is $\{0.252,0.211,0.316$, $0.291\}$.Since

$$
C R=0.00275<0.1 \text {, one consistency check is needed. }
$$

\section{CONCLUSIONS}

By applying the AHP virtual platform method to college student employment, we can understand the result straightforwardly. This is a simple, low-cost method and it can provide useful information and improve efficiency for those who are interested.

\section{ACKNOWLEDGEMENTS}

With students at the Department of mathematics teacher and thank the Science College of Northeast Dianli University!

\section{REFERENCES}

[1] Yang Bao-an, Zhang Ke-jing.A multi-objective decision analysis.Donghua UniversityPress,2012.

[2] Wang Lian-fen, Xu Shu-bai.An introduction to the analytic hierarchy process.China Renmin University Press,1990.6.

[3]Ding Jian,Wang Hua.A Brief Group Decision making Model of AHP and $a$ New Scale Method[J].management engineering (ME),2000,14(1):16 18.

[4]Zhao Chen, Xu Shu-bai .Analytic hierarchy process.Science press,1986(end).

[5]Shan Chen.On congestion control and fair bandwidth allocation in the internet.A Thesis Submitted to The Hong Kong University of Science and Technology in Partial Ful llment of the Requirements for the Degree of Doctor of Philosophy in Computer Science and Engineering. Hong Kong, July 2009. 40-59.

[6] Wang Gang, Dit-Yan Yeung, Frederick H Lochovsky. A kernel path algorithm for support vector machines. Department of computer science and engineering, Hong Kong University of Science and Technology, Clear Water Bay, Kowloon, Hong Kong, China, 2004. 10-52. 